\title{
Research on medical image segmentation technology
}

\author{
Wang Jinguo ${ }^{1, a}$, Wang $\mathrm{Na}^{2, b^{*}}$ (corresponding author), Wang Rui ${ }^{3, \mathrm{c}}$ \\ ${ }^{1}$ Department of Urology, the First Hospital of Jilin University,China \\ ${ }^{2}$ Department of Anesthesiology, the First Hospital of Jilin University, China \\ ${ }^{3}$ Department of Information Engineering, Jilin Business and Technology College. \\ awangjinguolily@163.com, ${ }^{\text {'}}$ lilyly12345@163.com,,Xiaoben6666@126.com
}

\begin{abstract}
Keywords: Medical image, Segmentation technology.
Abstract. Medical image technology, which is a new cross subject in recent years, has been studied in the research of medical image processing and image processing and analysis technology. It not only can greatly improve the clinical medical level, but also provides the digital realization method for medical research and education, such as: computer aided clinical operation, etc. It lays a solid foundation for medical research and development, has an inestimable value. This paper focuses on the analysis of medical image segmentation technology. Image segmentation is mainly used in the ROI (Region of Interest) extraction of the image features. The selection of ROI is actually the region segmentation which needs to be studied according to some feature of the image. How to use digital image processing technology to divide the ROI area reasonably and correctly is a difficult problem in medical image processing.
\end{abstract}

\section{Introduction}

Medical image has very important function in the process of medical diagnosis[1]. The doctor has a direct relationship with the understanding of the image information and the accuracy of the diagnosis. In the process of medical image acquisition and transmission, it is inevitable that the image quality can be reduced. How to restore the original image correctly is the main content of medical image processing. It is also the main content of medical image processing to extract useful information from the existing images by using the knowledge of computer graphics, statistics, and mathematics morphology and so on[2].

Image segmentation is to divide the image into regions with different characteristics and extract the technology and process of the object of interest[3]. It is a key step from image processing to image analysis. Image segmentation has been a hot topic in image processing. So far, there have been a variety of different types of segmentation algorithms[4]. Generally speaking, the image segmentation algorithm is generally based on two properties: one is boundary discontinuity between regions, the other is the similarity of the pixels in the region. The first nature of the application is based on the intensity of the discontinuous transformation of the image, such as the edge of the image. The main application of the second properties is to divide the image into a similar area, such as the threshold based image segmentation. In order to achieve the purpose of medical assistant diagnosis and surgical planning, the main task is to extract the organs, tissues or lesions which are extracted from the organs, tissues or lesions in the region of interest[5].

Although there are a lot of segmentation algorithms, more and more algorithms are proposed. However, there is not a universal algorithm can meet the application requirements of any situation. For the selection and determination of segmentation algorithm based on the characteristics of 
different segmentation and target image, we can achieve the best results by using various image segmentation techniques[6].

\section{Basic model of image segmentation}

Image segmentation is to use the given feature or a criterion to detect regions of consistency to reach the whole image is segmented into different regions, so that more high-level analysis and understanding possible. With the help of a set of concepts, the image segmentation can be defined as follows: setting the $R$ represent the entire image region, the $R$ segmentation can be viewed as $R$ into $\mathrm{n}$ meet the following five conditions are nonempty subset of $\mathrm{R}_{1}, \mathrm{R}_{2}, \ldots \ldots \mathrm{R}_{\mathrm{N}}$

(1) $\bigcup_{i=1}^{N} R_{i}=R$

(2) $R_{i} \mid \quad R_{j}=\Phi$

$i \neq j$

(3) $P\left(R_{i}\right)=T R U E$

$\mathrm{i}=1,2, \ldots \ldots, \mathrm{N}$

(4) $P\left(R_{i} \bigcup R_{j}\right)=F A L S E$

$\mathrm{i} \neq \mathrm{j}$

(5) $R_{i}$ is a connected area.

$\mathrm{i}=1,2, \ldots \ldots, \mathrm{N}$

The above five conditions have a clear meaning of mathematics. Condition (1) pointed out that the image segmentation results of all sub regions of the union should include all the pixels in the image, namely each pixel in the image have been assigned to a sub area. Condition (2) pointed out that the segmentation results are not overlapping each other, that is, a pixel can not belong to two regions at the same time. Condition (3) pointed out that each sub region of the segmentation result has the unique characteristics, and the same area of the pixel should have some same characteristics. Condition (4) pointed out that different sub regions have different characteristics, and the pixels in different regions also have different characteristics. Condition (5) indicates that the pixels in the same sub region of the segmentation result are connected, that is, any two pixels within the same sub region are connected with each other in the sub region.

\section{Segmentation algorithm and application based on gradient vector flow field}

There are two major limitations in the application of the traditional deformation model. One is the initial contour must be close to the target edge; otherwise the effect is not good. The other is when the crack or depression occurs in the boundary, the algorithm is also not good. In 1998, the concept of gradient vector flow field is put forward, which is used to replace the classical external force field, which can solve the above two major limitations of the traditional parametric deformation model.

The edge map of the image is defined:

$$
f(x)=-E_{e x t}(x, y)
$$

$\mathrm{E}_{\text {ext }}(\mathrm{x}, \mathrm{y})$ is an external potential energy function. For gray level images, if $I(x, y)$ is the gray for point $(x, y), E_{e x t}(x, y)$ is as following: 


$$
\begin{aligned}
& E_{e x t}(x, y)=-|\nabla I(x, y)|^{2} \\
& E_{e x t}(x, y)=-\left|\nabla G_{\sigma}(x, y) \cdot I(x, y)\right|^{2}
\end{aligned}
$$

In which, $G_{\sigma}(x, y)$ is a two-dimensional Gauss function, $\sigma$ is the standard deviation. The $\sigma$ value affects the contour segmentation effect to a great extent. The larger value of the image will become blurred boundaries, but the scope of help to expand the deformation profile. For two valued images, $E_{\text {ext }}(x, y)$ can be expressed as:

$$
\begin{aligned}
& \mathrm{E}_{\mathrm{ext}}(\mathrm{x}, \mathrm{y})=\mathrm{I}(\mathrm{x}, \mathrm{y}) \\
& E_{e x t}(x, y)=G_{\sigma}(x, y) \cdot I(x, y)
\end{aligned}
$$

The mapping of the boundary $\mathrm{f}(\mathrm{x})$ is calculated by the original image, which has the following features:

1. The gradient of $f(x)$ is $\nabla f$, which points to the edge of the image.

2. $\nabla f$ near the edge was larger, otherwise the value is smaller.

3. In the area of the gray average, $\mathrm{I}(\mathrm{x}, \mathrm{y})$ approximate at a constant, $\nabla f$ approximate at zero.

\section{Experimental results and analysis}

By Figure 1, we can see that the application of gradient vector flow field method, regardless of the initial contour is located within or beyond the real boundaries, the deformation of the contour has a larger range of action and good convergence, the final deformation after the iterative calculation is very close to the real boundary. For the gray images with noise, the gradient vector flow field method can obtain better segmentation results even if the image is not used to eliminate noise. The proposed method shows that the method has good robustness to image noise. These two advantages are that the gradient vector flow field model is very suitable for medical image segmentation.

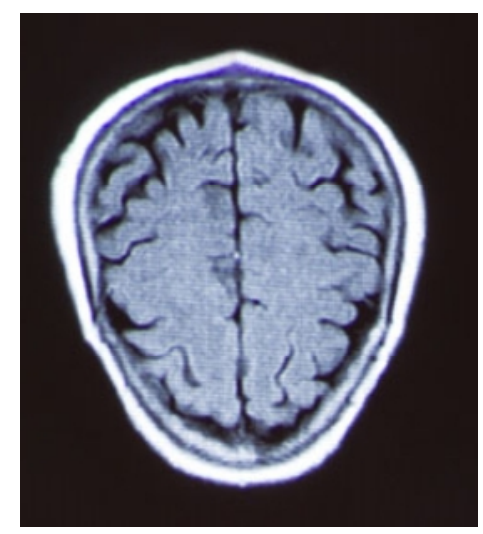

Figure 1. Cranial CD 


\section{References}

[1] Hussein R, Engelmann U, Schroeter A, et al. DICOM structured reporting Part 2. Problems and challenges in implementation for PACS workstations1[J]. Radiographics, 2004, 24(3): 897-909.

[2] Grauer D, Cevidanes L S H, Proffit WR. Working with DICOM craniofacial images[J].American Journal of Orthodontics and Dentofacial Orthopedics, 2009, 136(3): 460-470.

[3] Fernàndez-Bayó J, Barbero O,Rubies C,et al. Distributing Medical Images with Internet Technologies : A DICOM Web Server and a DICOM Java Viewer1[J]. Radiographics, 2000, 20(2): 581-590.

[4] Can A, H Shen, J N Turner, et al, Rapid Automated Tracing and Feature Extraction from Live High-Resolution Retinal Fundus Images Using Direct Exploratory Algorithms, IEEE Transactions on Information Technology in Biomedicine, 1999, 3(2): 125 138.

[5] M Sonka, M D Winniford, X Zhang, et al, Robust Simultaneous Detection of Coronary Borders in Complex Images, IEEE Transaction on Medical Images, 1995, 14(1): 151 161.

[6] McCormick B H, DeFanti T A, Brown M D, Visualization in Scientific Computing, Computer Graphics, 1987, 21(6): 249 266. 